\title{
Next generation sequencing in the clinical setting
}

\author{
Francesca Cortini ${ }^{1,2 *}$, Barbara Marinelli ${ }^{1}$ and Alessandra Bassotti ${ }^{3}$ \\ ${ }^{1}$ Department of Clinical Sciences and Community Health, University of Milan, IRCCS Ca' Granda Foundation, Italy \\ ${ }^{2}$ Genetics Laboratory, IRCCS Ca' Granda Foundation, Italy \\ ${ }^{3}$ Regional Center of Ehlers-Danlos Syndrome, IRCCS Ca' Granda Foundation, Italy
}

In recent years, Next Generation Sequencing (NGS) or deep sequencing have changed molecular approach in clinical laboratories. Before NGS advent, Sanger Method is based on sequencing one gene for one patient [1], it is laborious and cost-effective method. Whereas, by NGS applications it is possibly to sequence lots of genes and lots of patients in one experiment. There are different NGS applications to analyze all genes: Whole Exome Sequencing (WES) only proteincoding region are amplified; Whole Genome Sequencing (WGS) entire genome are amplified, Custom Target Sequencing regions include a particular group of genes (Target Approach) are amplified.

WES [2] and Custom Target Sequencing [3] become standard in clinical setting. Clinicians should be follow different steps to choose the right strategy: 1) disease model, 2) the region of interest, 3) depth of coverage (the average number of times that a particular nucleotide is present in a date position in a collection of random of sequences).

WES is an appropriate strategy for the identification of molecular defects in patients with suspected genetic disorders [2]. Moreover, WES is the best NGS method in case of conventional single gene sequencing or Custom panel are not identify the causal gene due to the genetic heterogeneity, atypical clinical presentation or lack of knowledge of the causal gene [4].

Custom Target Resequencing is the best NGS applications in case of the genes, related to disease, are known or genomic regions are characterized by bases repetition or CpG islands. The major advantage is the possibility to personalize the design (i.e. inclusion of complete gene sequence or specific intronic sequences) [3].
Up to date there are different platforms as MiSeq, NextSeq (Illumina), Ion Torrent (Thermo Scientific) to load NGS genomic libraries. These platforms generate millions of reads that are processed bioinformatically. Raw data, FastQ Files (file storing biological sequence and its quality score) are processed through their quality scores, then they are aligned to reference genome and each bases that composed reads are filtered on the base of statistics parametres and other kind of information to generate VCF files (Variant Calling Format Files, storing genetic variation data) [5].

The big challenge of clincal laboratories is to manage a huge amount of data produced by NGS. Moreover, it is imporatnt to standardize bioinformatics analysis and to make more easily accessible the NGS data interpretation.

\section{References}

1. Sanger F, Coulson AR (1975) A rapid method for determining sequences in DNA by primed synthesis with DNA polymerase. J Mol Biol 94: 441-448. [Crossref]

2. Yang Y, Muzny DM, Reid JG, Bainbridge MN, Willis A, et al. (2013) Clinical wholeexome sequencing for the diagnosis of mendelian disorders. N Engl J Med 369: 15021511. [Crossref]

3. García-García G, Baux D, Faugère V, Moclyn M, Koenig M, et al. (2016) Assessment of the latest NGS enrichment capture methods in clinical context. Sci Rep 6: 20948. [Crossref]

4. Alazami AM, Patel N, Shamseldin HE, Anazi S, Al-Dosari MS, et al. (2015) Accelerating novel candidate gene discovery in neurogenetic disorders via wholeexome sequencing of prescreened multiplex consanguineous families. Cell Rep 10: 148-161. [Crossref]

5. Danecek P, Auton A, Abecasis G, Albers CA, Banks E, et al. (2011) The variant call format and VCFtools. Bioinformatics 27: 2156-2158. [Crossref]
Copyright: (C2017 Cortini F. This is an open-access article distributed under the terms of the Creative Commons Attribution License, which permits unrestricted use, distribution, and reproduction in any medium, provided the original author and source are credited.
Correspondence to: Cortini Francesca, Phd, Department of Clinical Sciences and Community Health, University of Milan, Via San Barnaba 8, 20122, Milan, Italy, Tel: +39 02-55032433, Fax: +39 02-55032353, E-mail: francesca.cortini@ guest.unimi.it

Received: October 06, 2017; Accepted: October 25, 2017; Published: September 29,2017 